Review

\title{
Essential of Hair Care Cosmetics
}

\author{
Aurora Alessandrini * and Bianca Maria Piraccini \\ Division of Dermatology, Department of Experimental, Diagnostic and Specialty Medicine, \\ University of Bologna, Bologna 40138, Italy; biancamaria.piraccini@unibo.it \\ * Correspondence: aurora.alessandrini@alice.it; Tel.: +39-051-214-3677; Fax: +39-051-214-4867
}

Academic Editors: Won-Soo Lee and Enzo Berardesca

Received: 18 July 2016; Accepted: 20 September 2016; Published: 27 September 2016

\begin{abstract}
Nowadays, hair care and style play a very important role in people's physical aspect and self-perception. Hair cosmetics can be distinguished into two main categories: cosmetics with temporary effect on the hair, for example shampoos, conditioners, sprays, and temporary colors; and cosmetics with permanent effect on the hair, such as permanent waves, relaxers, bleaches and permanent colors. These cosmetic procedures may induce hair abnormalities. We provide an overview on the most important characteristics of these procedures, analyzing components and effects on the hair. Finally, we evaluated new camouflage techniques and tattoo scalp.
\end{abstract}

Keywords: hair; hair care; hair care cosmetics; hair care products; cosmetic ingredient; healthy hair

\section{Introduction}

Hair is a complex structure composed by many components that act as a unit, with the biological purpose of protecting the scalp, as well as physical attractiveness to the self-perception of beauty. Nowadays, hair care and style play a very important role in people's lives, both for men and women, so knowledge of hair products, mode of action, efficacy, ingredients and hair procedures has become more relevant in dermatologists' medical practice. The amount of money spent to enhance the hair beauty is an indication of how much attention is given today to the hair appearance. On the other hand, these data are emphasized in patients suffering from hair disease.

Hair cosmetics are also an important tool for increasing patient's adhesion to scalp treatments, according to the diversity of hair types and ethnicity.

Trueb [1] described them as "preparations intended for placing in contact with the hair and scalp, with the purpose of cleansing, promoting attractiveness, altering appearance, and/or protecting them in order to maintain them in good condition".

Hair cosmetics can be distinguished into two main categories:

(1) Cosmetics with temporary effect on the hair, for example shampoos, conditioners, sprays, and temporary colors; and

(2) Cosmetics that produce permanent effect on the hair shaft, such as permanent waves, relaxers, bleaches and permanent colors.

\section{Shampoos}

Shampoos are the most commonly prescribed treatment for hair and scalp. In the past, soap was the only available cleanser for the hair, while the introduction of the first non-alkaline shampoo date back to 1933 [2].

The modern society requires many features in a formulation: a shampoo has primarily to clean the scalp and the hair, but also has to be cosmetically pleasing, not dry out the hair, not irritate the skin, improve hair beautification, and be less expensive. 
A shampoo consists of 10-30 ingredients [2], classified according to their different activities. These ingredients include: (1) cleansing agents, called surfactants, that remove sebum or skin scale; (2) conditioning agents that give softness to the hair; (3) active ingredients, for treating specific disease s like dandruff; and (4) additives, such as preservatives, that contribute to the stability and comfort of the product. Every ingredient is tested, officially approved and declared on the label.

Thanks to surfactant agents, a shampoo can remove dirty particles and sebum from the scalp and from the hair shafts, but this cleansing activity has to be mild in order to avoid complete removal of the natural oils and sebum from the skin. Modern shampoos are sensitive on skin or mucous membranes, and the development of contact dermatitis is rare, due to the short contact time of the shampoo with the scalp. Typical ingredients of shampoos are foaming agents and detergent agents, which prevent the formation of insoluble soap on hair and scalp. Surfactant agents are the most important cleaning substances of shampoos, thanks to their molecular structure. In particular, one end of the molecule has a negative electrical charge and is soluble in water, so it does not blend with oils. The other end is soluble in oil and grease and does not blend with water. These molecules surround the fragments of grease localized on hair and scalp: the oil soluble parts go into the grease and the water-soluble parts remain located outward, forming a hydrophilic mass that is totally negatively charged. The surface of the hair shaft has negative charges, which tend to force the mass negative charges apart, resulting in a reduction of tension between water and grease on the surface, and production of foam, which incorporates the dirt, so that it cannot be re-deposited on the hair and scalp.

Conditioning molecules contained in shampoos combine the cleansing action with the function to impart manageability, gloss and antistatic properties of the hair. This conditioning shampoos are composed by fatty substances, like vegetable oils, wax, lecithin an lanolin derivatives, collagen, animal proteins, quaternium ammonium compounds or cationic polymers (which are the main component of the " 2 in 1 shampoo") and silicone, that reduce the friction obtained by combing the hair, and then the risk of hair shaft damage [2].

Among other substances we can mentioned: glycerin, polyvinylpyrrolidone, propylene glycol and stearalkonium chloride [3]. In particular, protein-derived substances have the property to be attractive by the keratin ("substantivity"), with the result of temporarily adhere the cortex fragments together. Additives of shampoos include ingredients that are able to control the thickness of the formulation like citrate and lactate.

Another key consideration is shampoos $\mathrm{pH}$ : many of them have an alkaline $\mathrm{pH}$, which causes hair shaft swelling, predisponding the hair to the damage. A neutral $\mathrm{pH}$ shampoo is the best choice for chemically treated hair from either permanent dyeing or permanent waving [3].

Other additives include UV absorbers, like benzophenone derivatives and antioxidants, like ascorbic acid and $\alpha$-tocopherol. Preservatives of shampoos prevent bacterial contamination, and include sodium benzoate, parabens, ethylenediaminetetraacetic acid (EDTA), DMDM hydantoin and tetrasodium EDTA.

Moreover, most shampoos contain other ingredients like colors, perfumes, pearlishing agents, moisturizers such as natural oils and fatty acid esters, and humectants, such as glycerin and sorbitol.

"Medicated" shampoos contain active ingredients selected to treat medical conditions, mainly seborrheic dermatitis or psoriasis. Active ingredients include ketoconazole, ciclopirox olamine, zinc pyrithione, piroctone olamine, tar derivates, salicylic acid, selenium sulfide, poly-vinyl-pyrrolidone, iodine complex, menthol and colloidal sulfur. The combination of cosmetic technology and medical therapy allows the benefits of cosmetic products together with the efficacy of medical agents.

Adverse reactions to shampoos are rare. The most common one is accidental contact with the mucous membranes, such as the nose and eye.

If patch testing to a shampoo is required, the shampoos should be diluted to form a $1 \%-2 \%$ aqueous solution for closed patch testing and a 5\% aqueous solution for open patch testing. False positive reactions due to irritation can occur. Patch testing individual ingredients separately is advisable [4]. 


\section{Conditioners}

Most of the shampoos contain conditioning agents, but consumers often apply a conditioner after shampooing, to minimize hair frizzing, increasing manageability and gloss of the hair. A conditioner can therefore be used to reduce a chemical or mechanical trauma of the hair, such as permanent dyes, bleaching, and excessive brushing.

As already mentioned, the hair shaft has negative electrical charges, while conditioning agents have positive charges: conditioners acts by neutralizing the electrical negative charge of the hair fiber by addicting positive charges and by greasing the cuticle, with the final result of reduce fiber hydrophilicity [5]. Conditioners also help the raised cuticles to lie down against the hair surface, and in this way preserve hair color and enhance its shyness and smoothness too.

Common ingredients of conditioners include mainly cationic surfactants, like cetyltrimethylammonium chloride, behentrimonium, propyltrimonium, and stearamidopropyl dimethylamine.

Polymers, like mono and polypeptides like hydrolyzed proteins (amino acids), polypeptides derived from collagen and polyvinylpyrrolidone (PVP), fill the hair shaft defects, creating a smooth surface to increase shine while eliminating static electricity thanks to its cationic nature [3].

Emollients/oily compounds are composed of natural or synthetic oils, but also esters and waxes. Jojoba oil, olive oil, or grape seed oil are some of these natural oils.

The most frequently used synthetic and active oils are silicones (e.g., dimethicone, dimethiconol, amodimethicone and cyclomethicone). Dimethicone is the most widely used silicone in hair care cosmetics, with the effect of protecting the hair shaft from abrasive actions.

Other conditioners components are: fatty alcohols (e.g., cetyl alcohol and stearyl alcohol), waxes (e.g., carnauba wax and paraffin wax), gums (e.g., guar gum), salt (sodium chloride) and emulsifiers like ethoxylated fatty alcohols (e.g., polysorbate-80 or ceteareth-20) [6].

In daily practice, hair conditioners can be used in different ways.

Instant conditioners are applied immediately after shampooing and rinsed off after few minutes, while deep conditioners, formulated usually in creams, can remain on hair for 20-30 min with an increase of their penetration.

Blow drying lotions are another formulation that can be left on the hair because are oil-free. They are very useful for people with fine hair or excessive scalp sebum. A particular type of conditioner, called hair glaze or hair thickeners conditioners, increase hair diameter by coating the hair shaft. Similar to conditioners, they usually contain proteins.

\section{Hair Color Products}

\subsection{Hair Dyes}

In modern society, people want to change the hair color according to the fashion trends of the moment, and thanks to developments in the field of dyestuffs, now this desire can be obtained in a few minutes. Nowadays, hair dyes are utilized by both men and women to modify natural hair color or restore pigmentation once graying has set in.

Modern hair dyes can be classified into three categories: temporary, semi-temporary and permanent. The main difference between them is their capacity to reach the hair cortex, obtained by an alkaline $\mathrm{pH}$. In many of these products, ammonia or ethanolamine are addicted to increase the $\mathrm{pH}$ [5].

Temporary colors are applied in the form of shampoos, lotions or foam and change the hair color for a relatively short time, normally until the first wash. They are generally used to add color highlights, remove yellowish hues from white hair, or cover up few graying hairs.

Ingredients of temporary colors are products with a high molecular weight, which simply deposit on the cuticle of the hair and are then removed easily. The dye is bonded to a cationic polymer to reduce its solubility and increase its affinity to hair. The resulting complex is dispersed in a base using surfactants to make the final product [7]. 
One example is henna, obtained from the plant Lawsonia alba, which is used on skin and nails, too. In the literature, few cases of allergic contact dermatitis to henna have been described [5,8].

Semi-permanent colors are composed by ingredients like nitroaromatic amines or aromatic dyes (of low molecular weight), which do not bind to the hair protein because they do not oxidize: they are water soluble, so they can be washed out after usually 6-10 wash cycles or earlier. They do not contain ammonia or ethanolamine, but hydrogen peroxide or resorcinol can be present. The main purpose of semi-permanent colors is to color white hair or to give more tone to the natural color, but they cannot lighten hair. One important characteristic is low molecular weight, which enables these products to diffuse into the middle layers of the cuticle without binding firmly to the hair protein itself [9]. Consequently, the application of semi-permanent colors does not require any prior hair modification, required instead by permanent hair color.

Permanent hair dyeing is a process that permanently changes the color of the hair, through a chemical reaction of oxidation, which allows the molecules of color to penetrate into the hair. Permanent colors allow darkening or brightening hair color, and are resistant to any external factor, including washings, for 4-6 weeks. They offer many range of hues and can cover the highest gray-hair numbers.

The principle consists in the penetration of the coloring molecules into pores of hair shaft (previously increased in size, by hydration and alkalinization). At this point, the molecules are oxidized and assume color, which is transmitted to the keratin of the cuticle and the cortex, mimicking natural melanin granules.

During these reactions, some intermediates are formed, usually derivates of $p$-phenylenendiamine (PPD), responsible for dark brown and black shades, or of $p$-aminophenol, responsible for blonde colors. Some dyes contain also resorcinol, but many companies have eliminated it for security reasons. PPD is an hapten (an incomplete antibody stimulating substance), which can combine with a protein molecule in the skin, leading to sensitization, which in future reapplication will lead to an allergic reaction. Allergic skin reactions to PPD may have different severity and sometime include the mucosae. For these reasons, a skin allergy test, made at least 48 hours before coloring, is mandatory before applying the coloring agent on the scalp in people with suspected PPD sensitization.

As mentioned, in dyes active ingredients are also present, like hydrogen peroxide, which bleaches out the original color and, oxidizing the molecules of the dye, activate the colorant reactions, and ammonia, which is the alkalizer. Other ingredients are: chelating agents, which maintain chemically stable peroxideand its activity, deactivating any catalytic metals in the product; and solvents, which help to improve viscosity and solubilization of the dye; surfactants, which remove oil and sebum from the hair surface.

Dyes can interfere with some dermoscopic diagnosis of hair diseases [10]: for example, permanent dye can penetrate hair follicle, giving a "black dot" appearance, distinctive for alopecia areata; semipermanent dye can deposit on the scalp, mimicking sun exposure or excoriated lesions.

Long-term contact of dyes with the scalp can determine a contact dermatitis which can cause a telogen effluvium (Figure 1), but in severe cases can induce facial edema.

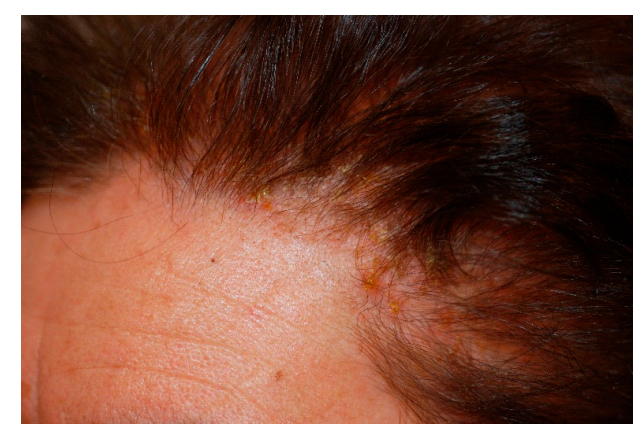

Figure 1. Contact dermatitis of the scalp due to permanent dye. 
As mentioned, PPD is an extremely potent contact sensitizer, allowed at a concentration up of $6 \%$ in hair dyes. Other frequent reported hair dye allergens are toluene-2,5-diamine, allowed for use at a concentration up to $10 \%, p$-aminophenol and $m$-aminophenol, the last at up to $2 \%$ [11].

A study [11] analyzed chemically the actual content of PPD and selected derivatives in hair dye products causing allergic contact dermatitis in nine patients: more specifically, chemical analysis of the randomly collected products identified concentrations of the oxidative hair dyes similar to the concentrations in the products used by the patients with hair dye dermatitis. This study shows that patients can develop hair dye dermatitis from concentrations lower than that permitted in the European Union (EU) Cosmetic Directive.

In another interesting study [12], authors provided a ranking concerning contact allergy risk, analyzed the predictions for sensitization potential of 229 hair dyes substances: as result, about the $75 \%$ of all hair dyes were predicted to be strong/moderate sensitizers, even stronger than PPD, and many of these are not currently used for diagnosing contact allergy to hair dyes. PPD is resulted more potent than toluene-2,5-diamine, while resorcinol seems to be less potent than PPD. Only $22 \%$ of hair dye substances were predicted to be weak sensitizers and 3\% were predicted to be extremely weak or non-sensitizing.

More recently, Uter et al. [13] assembled complete information on the current practice of using hair cosmetics, creating a new European hair cosmetics series. They collected all allergens of proven importance for patients with contact allergy to hair cosmetics, and also allergens with less proven importance, underlining the need to evaluate these substances in specialized departments, and to create a cosmetovigilance network.

\subsection{Bleaching Agents}

These products provide permanent hair lightening without the addition of a coloring tint, thanks to substances like hydrogen peroxide, ammonia and persulfate salts. The process requires time and is realized by a series of color stages. As mentioned, hydrogen peroxide bleaches out the original color of the hair. The oxidizing agent, in alkaline solution, penetrates into the cortex of the hair, after raising the scales of the cuticle, and chemically modifies the melanin: as result, melanin granules are dissolved, leaving tiny gaps in the cortex [7]. For this reasons, repeated bleaching treatments can cause an increased porosity of hair and permanently raised scales, leading to hair weathering (Figure 2).

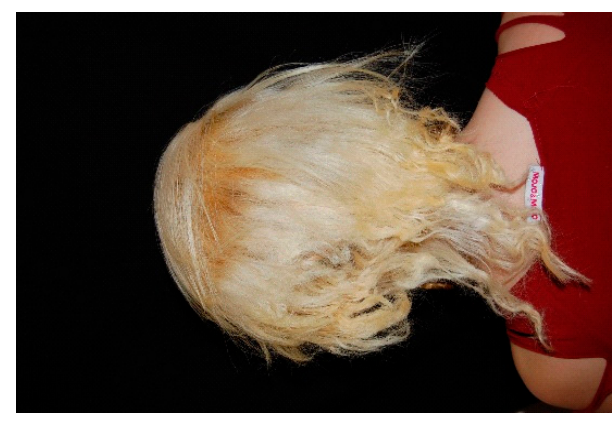

(a)

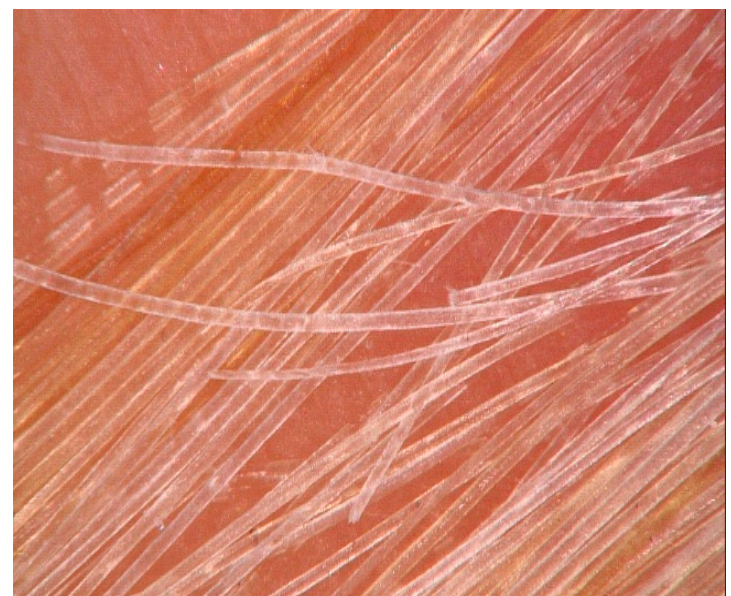

(b)

Figure 2. (a) Hair weathering caused by repeated bleaching treatment; and (b) important hair shaft damage of hair weathering, clearly visible with microscopic examination.

Persulfates can trigger immunomediate reactions, such as rhinitis, asthma, contact urticarial and even anaphylaxis, but the exact immune mechanism is still unknown [14]. 


\section{Hair Styles}

\subsection{Permanent Waves}

The topical application of cosmetics in lotions or sprays may give hair a temporary different "style" that is lost after the first shampooing. A modification of hair internal chemical structure with a specific chemical process is needed to change in a permanent way the shape of the hair.

A permanent wave is a process that makes curly the hair, using chemicals that break and reform the strong disulfide bonds of the hair shaft. First the hair is washed and wrap around the curler (the amplitude of which will determinate the thickness of the curl), then waving lotion in alkaline base is then applied (the most commonly used is ammonium thioglycolate): it raises the scales of the cuticle and, reaching inside the cortex, breaks the disulfide bonds that give the natural shape of the hair. After a fixed time the waving lotion is removed with the rinse and a neutralizer is applied, which contains oxidizing agents, like hydrogen peroxide, that rebuild the disulfide bonds in a different order, stabilizing the new shape of the hair. Hair waving is for this reason the most dangerous cosmetic procedures for the hair and should be monitored carefully.

Trichorrhexis nodosa is frequent in bleached and permed hair. The damage to the cuticle cells makes them detach and lead to exposure to the hair shaft cortex. The hair shaft shows small whitish knots visible with the unaided eye. The hair is fragile and short due to breakage. Microscopic observation of the hair shaft shows one or more knots that easily break off producing two brush-like tips (Figure 3).

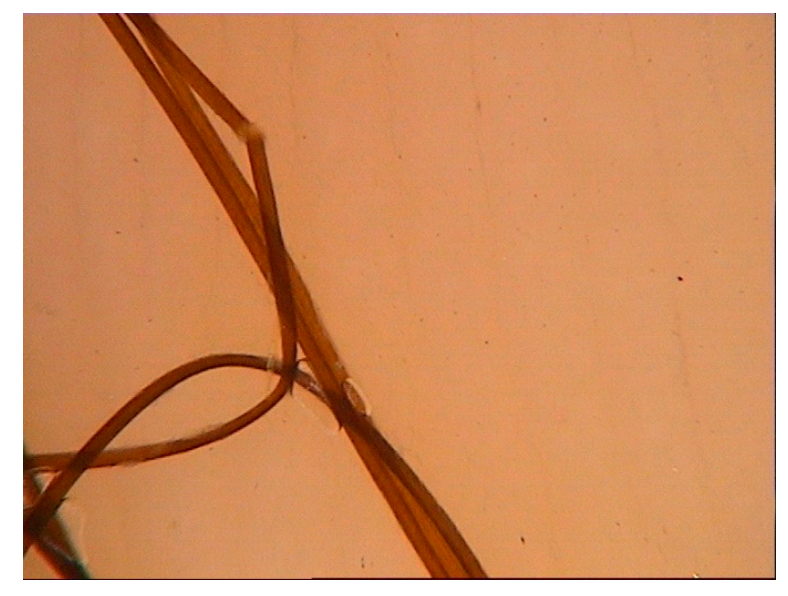

Figure 3. Multiple Knots easily break off, producing two brush-like tips, are typical hair shaft abnormalities of trichorrhexis nodosa.

\subsection{Relaxers}

The mechanisms of relaxing is the breaking of hair disulfide bonds, re-forming of hair shape and re-making of the linkages. The shape of the hair changes from curly to straight. Multiple procedures or prolonged time of exposure to the relaxer may be needed to straighten tightly curled hair, such as that of African-Americans, producing hair weathering and breakage.

\subsection{Hair Straightening}

In the past, hair straightener procedure consisted on applying petrolatum based oils on the hair combined with hot irons or hot combs pressed to the hair, but the effect was only temporary.

Nowadays, hair straighteners are called chemical relaxers, and the effect of hair straightening is permanent. Thanks to the high $\mathrm{pH}(9.0-14.0)$, the solution inflate the hair, opening cuticle scales, which allows the alkaline agent $\left(\mathrm{OH}^{-}\right)$to penetrate into the hair fibers up to the endocuticle. The straightening 
product reacts with keratin, breaking and rearranging the disulfide bridges, which making the spiral keratin molecule soft and stretched [5]. This procedure needs to be repeated every 12 weeks or longer.

Alkaline straighteners contain 1\%-10\% sodium hydroxide (lye-relaxer), lithium hydroxide, calcium hydroxide or a combination of these ingredients such as guanidine carbonate and calcium hydroxide (no-lye relaxers). Ammonium thioglycolate is another "no-lye" relaxers, which selectivity weakens the hair cysteine bonds: it is then oxidized by hydrogen peroxide and, applying a hot iron during the process, the hair straightening can be obtained. Thyoglycolate and hydroxides are not mutagenic or carcinogenetic. The main adverse effects of hair straightening are scalp burns and hair breakage. In particular, in the study of Shetty et al., the most common adverse effects reported after chemical hair straightening were: frizzy hair in $67 \%$, dandruff in $61 \%$, hair loss in $47 \%$, thinning and weakening of hair in $40 \%$, greying of hair in $22 \%$, and split ends in only $17 \%$ [15].

A keratin treatment containing formaldehyde, a well-known carcinogen has been performed in Brazil with success: soon the health vigilance organizations in Brazil (Agência Nacional de Vigilância Sanitária) prohibited the use of any product containing formaldehyde in concentrations above $0.2 \%$ for cosmetics [16]. Afterward, the formaldehyde was replaced by a potentially more mutagenic and neurotoxic product, glutaraldehyde, which belong to the same aldehyde group, sold as a homemade treatment. The ingredients used today do not contain any of these two substances, but are based on formaldehyde-releasers such as methylene glycol or glyoxylic acid. Acute contact dermatitis may occur as adverse effect in previously sensitized patients [16].

\section{Hair Extensions}

Hair extensions have become worldwide increasingly popular, either as a beauty accessory for healthy people both as an aesthetic aid for patients with hair loss.

They are composed by synthetic or human hair, which can be attached to natural hair with various techniques like strand-by-strand, the weft or the clip-in methods. The cuticles of the extensions must be aligned in the same direction of the natural hair and they are usually no longer than twice the length of the person's natural hair for a cosmetically harmonious result.

Among local side effects, we can mention traction alopecia, secondary to the pulling force applied to the hair follicle over time [17] (Figure 4), pruritus, tenderness, and local pain. Hair matting, which describes reversible or irreversible tangling of locks of hair that stick together, can occur [18] (Figure 5).

Allergic contact dermatitis or contact anaphylaxis can arise after sensitization to glues or natural rubber latex that is incorporated into the adhesive for hair extensions.

Trichobezoars formed by the consumption of hair extension is a rare cause of Rapunzel syndrome, which requires surgery and psychiatric examination $[19,20]$.

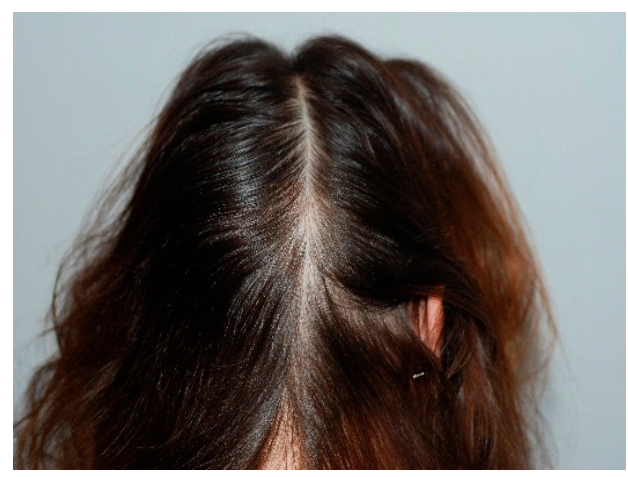

(a)

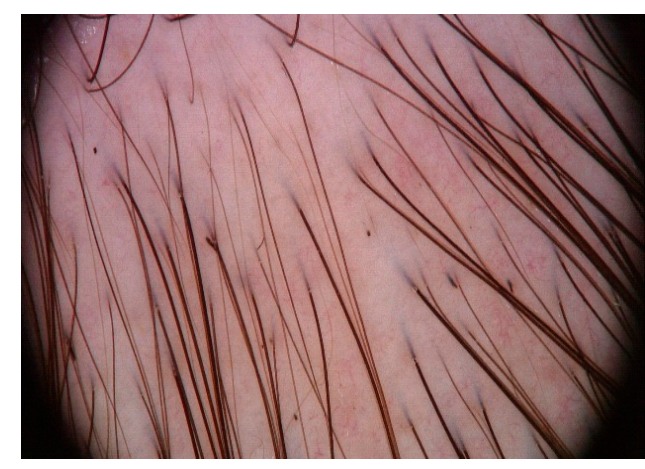

(b)

Figure 4. (a) Small alopecic patch at the site of attachment of previous hair extension; (b) Dermoscopic aspect of traction alopecia: multiple broken hairs in a non scarring patch of alopecia. 


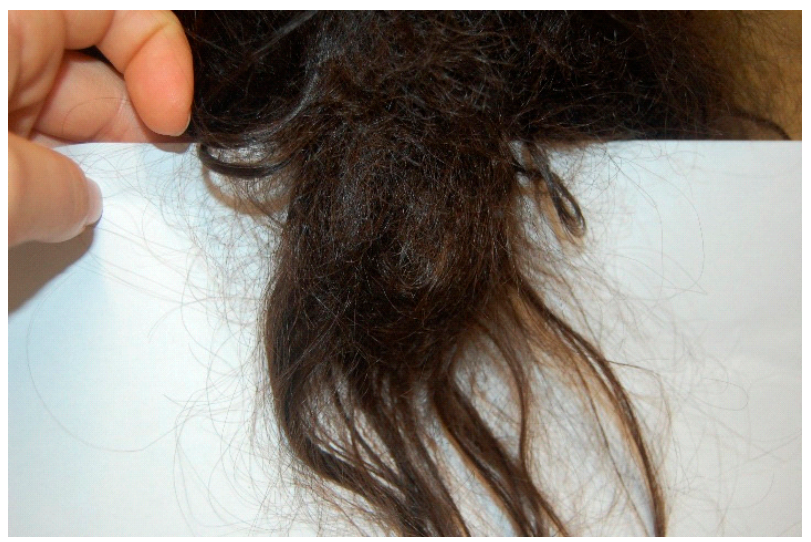

Figure 5. Hair matting developed at the site of attachment of extensions.

A case of homicidal strangulation by victim's own artificial hair extensions has also been described [21].

\section{Camouflage and Tattooing}

People experiencing alopecia undergo emotional distress from their condition, but fortunately many new techniques have been developed to hide some hair disease.

Patients affected by alopecia areata can cover the scalp using many different styles of hairpieces, which are made with human or synthetic hair fibers tied or woven to a fabric base. In particular, human hair wigs are the most expensive, and they need to be washed and styled every two weeks, lasting 2-3 years. Synthetic wigs are stronger and easier to maintain. Types of hairpieces include: wig, demiwig, toupees, fall, cascade and wiglet [22]. Allergic contact dermatitis due to incorporated glues or natural rubber latex car occur (Figure 6).

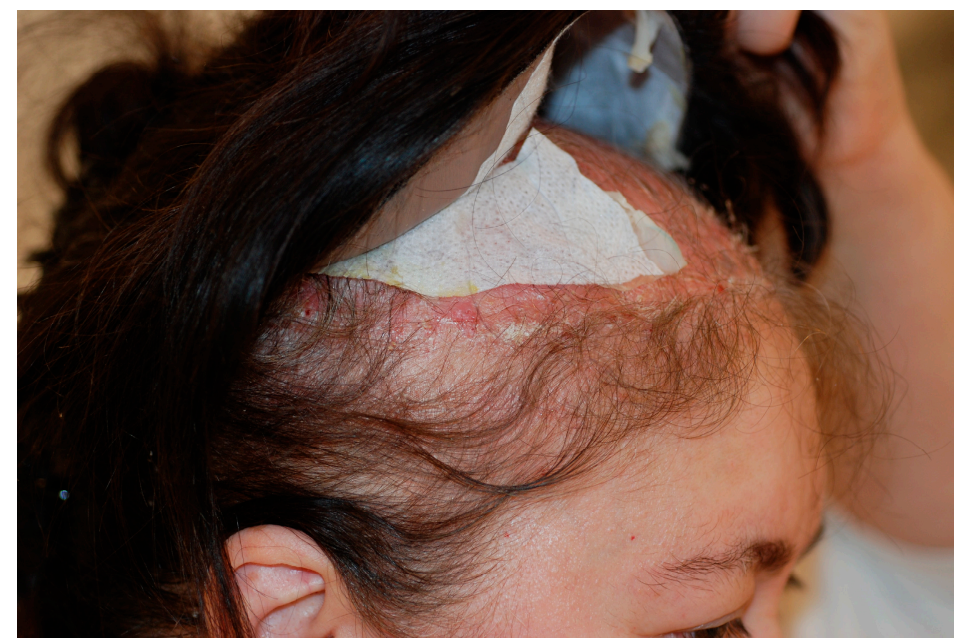

Figure 6. Severe contact dermatitis of the scalp due to wig glue.

Pigmented hair and thickening fibers are effective to cover scalp alopecia in patients with epidermolysis bullosa [23] or androgenetic alopecia [24].

They act bonding to the hair by static electricity, so it appear thicker. A fixative spray applied on fibers can increase their stability. Pruritus and irritation and skin irritation can occur.

A split or flattened hair direction around the parietal whorl (PW) can be cosmetically unpleasant. A scalp medical tattooing technique can camouflage this problem, using a jet black color ink of commercial products, with high satisfaction of the patient and no complications [25]. 
A tattoo technique, called scalp micropigmentation (SMP), has been recently developed to mimic hair follicle and minimize cosmetic problem derived from hair diseases. It offers good solution for patient with scarring alopecia, alopecia areata or alopecia totalis, chemotherapy patients, people with scalp deformities and many other problems [26].

SMP is a permanent concealer, which use pigments of different hues in a variety of formulas and viscosities and instruments employing needles in various sizes and shapes. Many factors have to be evaluated when performing this technique, like aesthetic factors, presence of scars on the scalp, skin color, hair color, the amount of remaining hair, and the color and viscosity of the pigments used.

The SMP process begins by inserting a micro droplet of pigment through the skin and into the upper dermis, using a standard tattoo instrument, which supports between one and six needles cycling between 100 and 150 cycles per second. Multiple sessions are needed to complete the procedure.

The operator makes technical and artistic judgments as the process advances, millimeter by millimeter, customizing each treatment on the patient.

Risks and complications include infection, allergies to the components in the pigments and magnetic resonance imaging complications, such as swelling and pain from the metals in the pigments.

Little is known about the chemical composition, adsorption, distribution, metabolism, and excretion of inks, and other studies are needed to learn more about this procedure.

\section{Conclusions}

Hair styling play a very important role in people's physical aspect and self-perception. Hair cosmetics is a very interesting evolving field, and hair industry is costantly investing resources to find new components, molecules and hair procedures. Usually patients with hair disorders are the main actors targeted to these products, but in daily life all people care of hair beauty and health.

Dermatologists should always be update on new formulations, hair technique or adverse effects of hair products designed to beautify and modify the hair, in order to better treat hair and scalp contiditions.

Author Contributions: The Authors contribute equally to this work.

Conflicts of Interest: The authors declare no conflict of interest.

\section{References}

1. Trüeb, R.M. Dermocosmetic aspects of hair and scalp. J. Investig. Dermatol. Symp. Proc. 2005, 10, $289-292$. [CrossRef] [PubMed]

2. Trüeb, R.M. Shampoos: Ingredients, efficacy and adverse effects. J. Dtsch. Dermatol. Ges. 2007, 5, 356-365. [CrossRef] [PubMed]

3. Draelos, Z.D. Shampoos, conditioners, and camouflage techniques. Dermatol. Clin. 2013, 31, $173-178$. [CrossRef] [PubMed]

4. De Groot, A.C.; Weyland, J.W.; Nater, J.P. Unwanted Effects of Cosmetics and Drugs Used in Dermatology; Elsevier: Amsterdam, The Netherlands, 1994; pp. 473-476.

5. Dias, M.F.R.G. Hair cosmetics: An overview. Int. J. Trichology 2015, 7, 2-15. [CrossRef] [PubMed]

6. Souza, P.; Rathi, S.K. Shampoo and conditioners: What a dermatologist should know? Indian J. Dermatol. 2015, 60, 248-254. [PubMed]

7. Guerra-Tapia, A.; Gonzalez-Guerra, E. Hair cosmetics: Dyes. Actas Dermosifiliogr. 2014, 105, $833-839$. [CrossRef] [PubMed]

8. Thorén, S.; Yazar, K. Contact allergens in 'natural' hair dyes. Contact Dermat. 2016, 74, 302-304. [CrossRef] [PubMed]

9. Neuser, F.; Schlatter, H. Hair dyes. In Cosmetic Dermatology: Products and Procedures; Draelos, Z.D., Ed.; Wiley-Blackwell: Oxford, UK, 2010; pp. 256-310.

10. Angra, K.; LaSenna, C.E.; Nichols, A.J.; Tosti, A. Hair dye: A trichoscopy pitfall. J. Am. Acad. Dermatol. 2015, 72, e101-e102. [CrossRef] [PubMed] 
11. Søsted, H.; Rastogi, S.C.; Andersen, K.E.; Johansen, J.D.; Menné, T. Hair dye contact allergy: Quantitative exposure assessment of selected products and clinical cases. Contact Dermat. 2004, 50, 344-348. [CrossRef] [PubMed]

12. Søsted, H.; Basketter, D.A.; Estrada, E.; Johansen, J.D.; Patlewicz, G.Y. Ranking of hair dye substances according to predicted sensitization potency: Quantitative structure-activity relationships. Contact Dermat. 2004, 51, 241-254. [CrossRef] [PubMed]

13. Uter, W.; Bensefa-Colas, L.; Frosch, P.; Giménez-Arnau, A.; John, S.M.; Lepoittevin, J.P.; Lidén, C.; White, I.R.; Johansen, J.D. Patch testing with hair cosmetic series in Europe: A critical review and recommendation. Contact Dermat. 2015, 73, 69-81. [CrossRef] [PubMed]

14. Hougaard, M.G.; Menné, T.; Søsted, H. Occupational eczema and asthma in a hairdresser caused by hair-bleaching products. Dermatitis 2012, 23, 284-287. [CrossRef] [PubMed]

15. Shetty, V.H.; Shetty, N.J.; Nair, D.G. Chemical hair relaxers have adverse effects a myth or reality. Int. J. Trichology 2013, 5, 26-28. [CrossRef] [PubMed]

16. Van Lerberghe, L.; Baeck, M. A case of acute contact dermatitis induced by formaldehyde in hair-straightening products. Contact Dermat. 2014, 70, 384-386. [CrossRef] [PubMed]

17. Salibi, A.; Souéid, A.; Dancey, A. Hair braiding (plaiting) and hair extensions: An underestimated danger! J. Plast. Reconstr. Aesthet. Surg. 2014, 67, e206-e207. [CrossRef] [PubMed]

18. Yang, A.; Iorizzo, M.; Vincenzi, C.; Tosti, A. Hair extensions: A concerning cause of hair disorders. Br. J. Dermatol. 2009, 160, 207-209. [CrossRef] [PubMed]

19. Flaherty, D.C.; Aguilar, F.; Pradhan, B.; Grewal, H. Rapunzel syndrome due to ingested hair extensions: Surgical and psychiatric considerations. Int. J. Surg. Case Rep. 2015, 17, 155-157. [CrossRef] [PubMed]

20. Kwong, W.T.; Kalmaz, D. A modern form of Rapunzel syndrome: Trichobezoar composed of synthetic hair extensions. Clin. Gastroenterol. Hepatol. 2014, 12, A33-A34. [CrossRef] [PubMed]

21. Al Beraiki, K.H.; Schiwy-Bochat, K.H.; Rothschild, M.A. Homicidal strangulation by victim's own artificial hair extensions. Int. J. Legal Med. 2011, 125, 459-461. [CrossRef] [PubMed]

22. Draelos, Z.D. Camouflage technique for alopecia areata: What is a patient to do? Dermatol. Ther. 2011, 24, 305-310. [CrossRef] [PubMed]

23. Harris, A.G.; Kim, M.; Murrell, D.F. Pigmented hair-thickening fibers: A camouflage technique for alopecia in patients with epidermolysis bullosa. Skin Appendage Disord. 2016, 1, 153-155. [CrossRef] [PubMed]

24. Cossman, J.P.; Ladizinski, B.; Lee, K.C. Pigmented concealing powders for the hair loss patient. J. Cosmet. Dermatol. 2013, 12, 322-324. [CrossRef] [PubMed]

25. Park, J.H.; You, S.H. Scalp medical tattooing technique to camouflage bifid parietal whorls. Plast. Reconstr. Surg. Glob. Open 2016, 4, e671. [CrossRef] [PubMed]

26. Rassman, W.R.; Pak, J.P.; Kim, J.; Estrin, N.F. Scalp micropigmentation: A concealer for hair and scalp deformities. J. Clin. Aesthet. Dermatol. 2015, 8, 35-42. [CrossRef] [PubMed]

(C) 2016 by the authors; licensee MDPI, Basel, Switzerland. This article is an open access article distributed under the terms and conditions of the Creative Commons Attribution (CC-BY) license (http://creativecommons.org/licenses/by/4.0/). 